\title{
Revisión del trabajo de tesis
}

\section{Thesis work review}

\author{
OROZCO-OROZCO, José Zócimo*†
}

Universidad de Guadalajara, Centro Universitario de Ciencias Sociales y Humanidades, División de Estudios Jurídicos. Doctorado en Derecho. Av. Juárez No. 976, Colonia Centro, Guadalajara, Jalisco, México. C.P. 44100

ID $1^{\text {er }}$ Autor: José Zócimo, Orozco-Orozco

\section{Resumen}

Dentro del proceso de elaboración de una tesis profesional la acertada y meticulosa revisión por parte del director es esencial, tanto en la forma como en el fondo. Desde la elaboración del protocolo de investigación será invaluable su apoyo para definir el título de la tesis y localizar fuentes actuales. En la revisión, se hace una evaluación de cómo se realiza la corrección del original en forma profesional y el apoyo que debe tener el alumno para lograr elaborar tesis de calidad, incluyendo darle toda la información que le será muy útil al sustentar su examen de tesis. El director está al pendiente de los puntos más importantes que se deben unificar en la redacción del original y las fuentes para la corrección. Es muy valioso que no se pierdan las recomendaciones que hacen los integrantes del jurado de examen, por lo cual se propone que se integren al documento original.

\section{Revisión, Tesis, Trabajo de tesis}

\begin{abstract}
Through the process of making a professional Thesis, the revision of the elements of substance and form by the Director of Thesis is very important. Beginning with the Thesis protocol is very important the title of the Thesis and having current literature. In the stage of Review the correction of the original work and the support that is supposed to have the candidate for making a good quality work, and that includes giving information to pass the grade exam. The Director of Thesis should always supervise the most important points in the writing process and collect information resources. The recomendations gived by the exam jury are suggested to be integrated in the final Thesis work.
\end{abstract}

\author{
Revision, Thesis, Work of Thesis
}

\footnotetext{
* Correspondencia del Autor (josez_orozco@hotmail.com)

$\dagger$ Investigador contribuyendo como primer Autor.
} 


\section{Introducción}

El alumno debe realizar una investigación de tesis para sustentar el examen y obtener el título correspondiente a sus estudios efectuados en una institución educativa. El proceso de elaboración de dicho trabajo de investigación tiene varias etapas. Primero se hace el proyecto llamado protocolo de investigación y se redacta el original en varios capítulos, jerarquizados de acuerdo al tema. Cada uno de dichos pasos implica una revisión constante hasta que no aparezcan errores en la redacción y puntuación, en las citas, etc., tomando en cuenta que los errores que aparezcan después ya no podrán corregirse y serán motivo de evaluación.

\section{Justificación}

Es frecuente que una gran parte de las tesis que se presentan para obtener el título de estudios realizados tengan recomendaciones que hacen los integrantes del jurado. Debe ser un objetivo que el alumno se convierta en el principal revisor de su investigación, sin embargo, el director deberá tener el compromiso de sugerir acciones que auxilien a su tesista a cumplir esta meta. No es suficiente que el director sea experto en el tema, el dominio de la metodología de investigación será un valioso instrumento que aunado a su experiencia profesional contribuirá al total éxito y titulación del postulante.

En el examen profesional se mencionan entre otros problemas: errores de ortografía, todo lo que le faltó al documento, autores o teorías que era esencial su consulta y se ignoraron, errores en el trabajo de campo, en la determinación de la muestra, insuficiente obtención de información para la elaboración de cuadros y gráficas, etc. Una acertada revisión deberá evitar estas situaciones ante el jurado de examen. Las observaciones se deben agregar al documento original, ya que serán valiosas para otras investigaciones que se relacionen con el trabajo desarrollado.

\section{Elementos metodológicos Hipótesis}

Este trabajo orientará a quienes deseen ser profesionales en la revisión de trabajos de investigación obteniendo que se presenten tesis de calidad, por medio del fortalecimiento de la formación académica en la corrección de originales.

\section{Planteamiento del problema}

El alumno tiene dificultades para realizar su trabajo de tesis y su director debe apoyarlo con información que le permita conocer cómo ir elaborando cada capítulo y al final, hacer una buena corrección de su original de investigación.

\section{Objetivo general}

Dar a conocer los avances que debe saber el alumno para que mejore el contenido de su tesis por medio de la revisión.

\section{a) Objetivo particular}

Que se aplique la revisión del original de tesis como un instrumento indispensable en cada paso de la investigación, para eliminar errores y perfeccionar integralmente el documento que se vaya elaborando.

\section{b) Objetivo particular}

Que el alumno elabore tesis de calidad y se rescaten las recomendaciones que hacen los integrantes del examen de titulación como parte de la revisión de tesis.

\section{Metodología}

Se aplican conocimientos basados en la observación, en mi experiencia como profesor por más de cuatro décadas de impartir materias relacionadas con el proceso de tesis. La técnica de la investigación documental para dar a conocer información valiosa sobre el proceso de elaboración de una tesis en diversos niveles de estudios. Se utiliza el método deductivo para tratar el asunto de lo general a lo particular; el método científico, para hacer el planteamiento de un problema, el análisis y llegar a conclusiones y propuestas.

Por medio del método documental, se rescatan recomendaciones que hacen autores en el tema tratado. El método experimental se aplica en destacar los conocimientos adquiridos en la impartición de materias relacionadas con el proceso de investigación de tesis y que resultan valiosos, por los resultados obtenidos. 


\section{Importancia de la revisión del trabajo de tesis}

Una parte de la elaboración de tesis, consiste en revisar en forma constante el avance del trabajo de investigación. Debe hacerse una cuidadosa corrección durante este proceso y una vez terminada la investigación, realizar una revisión final muy cuidadosa del original. De la calidad de la revisión y corrección que se haga dependerá en gran parte el éxito del alumno en la evaluación de examen para obtener el grado correspondiente de estudios realizados.

Estamos hablando que esta actividad la hace el alumno en primer lugar, trabajo que es revisado por el director; actualmente también participan los sinodales de coloquios de avances de tesis, que se realizan cada semestre durante el tiempo que se cursa el programa de posgrado, conjuntamente con el maestro de la asignatura de Desarrollo de Tesis. Una vez concluido los estudios y elaborada en forma completa la investigación se efectuará una revisión por el alumno, el director y los lectores de tesis y ya en el examen, serán los sinodales los que harán una evaluación, pero ya no habrá espacio para hacer correcciones en el original de tesis, sólo anotar las recomendaciones aparte por el sustentante para futuros estudios o publicación en forma de libro.

Mi punto de vista es que las observaciones que hacen los sinodales deben quedar integradas a la tesis; deberían agregarse al original por el alumno, como se hacía en la Facultad de Turismo de la Universidad de Guadalajara anteriormente. Hoy, el hecho que el alumno lleve la tesis ya impresa al examen evita que queden consignadas las observaciones y sugerencias del jurado.

\section{¿Quién realiza el trabajo de revisión de tesis y qué señalamientos hace?}

a) El alumno. Busca ayuda de quién será su director, que le diga si su tema elegido está bien o algún otro tema que le recomiende. La mayoría de alumnos necesitan de ayuda metodológica para la elaboración de su protocolo o proyecto de tesis. Se debe nombrar a maestros que tengan tiempo de apoyarlos, pues la elección de un buen director que tenga tiempo para dedicarles será crucial para llegar a la titulación.
Una de las principales dificultades que tiene el alumno actualmente es la falta de habilidad para redactar, labor que deberá asumir el director, dando las directrices necesarias para llegar al objetivo. El alumno busca que esté completo el trabajo que le piden y esa es otra orientación del director, basado en su experiencia de metodología de la investigación. Ya en este punto se debe fortalecer el trabajo de revisión que hace el alumno sobre su investigación.

b) Evaluador de protocolo. Es un profesor que revisa que el documento de planeación de tesis esté completo; que el tema no haya sido ya aprobado por los legisladores; recomienda el acudir a más fuentes de consulta, bases de datos, organismos encargados de publicar información estadística especializada, por ejemplo; también que no tenga evidentes errores ortográficos. Se revisa que la hipótesis sea congruente con el trabajo y que la redacción sea clara. Hace recomendaciones para el alumno o rechaza el tema, es decir no lo aprueba.

c) El sinodal de coloquio de avance de investigación de tesis. Son importantes las recomendaciones por el sinodal, le permite al alumno mejorar su estructura y contenido de tesis. Estas orientaciones son muy útiles, porque serían los señalamientos que le harían al alumno en un examen profesional para obtener el título correspondiente.Las dificultades que tiene el evaluador es que a veces no le entregan el documento con suficiente tiempo de anticipación, para hacer una buena evaluación. En diez minutos que por lo general dura la exposición del alumno es insuficiente para que el sinodal pueda tener los elementos que le hacen falta para hacer buenas recomendaciones.

d) Maestro de la asignatura de Desarrollo de Tesis. Su materia consiste precisamente en apoyar a los alumnos en su avance de tesis. El profesor tiene un plan de cómo trabajar con sus alumnos y conocer lo que cada uno necesita. La asignatura tiene calificación y es posible avanzar en cada una de las investigaciones. 
e) Maestros de cada una de las materias del plan de estudios. Para conocer a sus alumnos, cada profesor, mínimo pregunta a cada uno ellos cuál es el título de su tesis y la hipótesis, con lo cual se entabla la conversación y dan su opinión. Esta participación de apoyo es durante el tiempo de estudios, pero viene la etapa final de revisión del trabajo académico, cuando el alumno termina sus estudios.

f) Director de tesis. Está con el alumno desde la elaboración de su protocolo. Primero le pide a su alumno que avance, luego que esté completa y hace correcciones al trabajo. Para el director de tesis es muy importante que el trabajo sea correcto, porque en el examen está de por medio su calidad de dirección de la investigación. (Rivera-Camino, Jaime, 2014, p. 105). Señala que no falte ninguna parte de las que se estipulan en la guía de titulación de la escuela o posgrado donde realiza sus estudios. Una vez que considera que está bien el trabajo de investigación extiende la carta de liberación de tesis para que alumno realice los trámites en su institución educativa.

g) Lector de tesis. Se asigna por lo general a dos maestros que dominan el tema y cada uno emite su dictamen por separado. Aquí es donde se detiene el proceso de titulación en una parte de los casos. Se hace una revisión estricta del contenido de forma y de fondo. Aunque se señala por lo general en los reglamentos de titulación de las instituciones educativas que las recomendaciones del lector quedan sujetas a la consideración del director, sí se respetan. Este trámite puede llevarse bastante tiempo por diversos motivos: el alumno no hace las correcciones con prontitud, tarda el director en apoyarlo o el lector no está de acuerdo con el trabajo de revisión realizado.

No obstante el apoyo que debe tener el alumno en el desarrollo de su trabajo de investigación, nos damos cuenta que hace falta resolver la ausencia de conocimientos metodológicos en el proceso de revisión de tesis $\mathrm{y}$ ese es el motivo de esta ponencia, que el investigador-alumno adquiera en primer lugar la responsabilidad de la revisión del trabajo de tesis, que sea un profesional.

\section{Conocimientos que el alumno debe adquirir en el proceso de revisión de tesis}

\section{Símbolos de corrección}

Es necesario que el alumno conozca los símbolos de corrección que se deben aplicar en su tesis, en su momento de revisarla y además que le serán muy útiles en estudios posteriores o cuando publique una ponencia o un libro y tenga que revisarlo ante una editorial. Dichos símbolos ayudan a simplificar el trabajo de corrección, permiten evitar anotar mucha información, bastará su señalamiento y se sabrá su significado por especialistas en una editorial. Además, hacer la corrección por el mismo investigador le ayudará a economizar en la presentación y publicación de sus trabajos. Esta tarea, considero no se ha atendido completamente para ayudar al alumno por parte de las instituciones educativas en la actualidad. (Ver cuadro No. 1).

\section{Cómo se hace la corrección de un original}

Se anota al final de la línea donde se encuentra la corrección una señal o asterisco para indicar que en ese lugar hay una corrección. También se puede para precisar más dicha corrección poniendo un símbolo donde está la errata y de esa manera se localiza fácilmente el lugar para hacer la modificación.

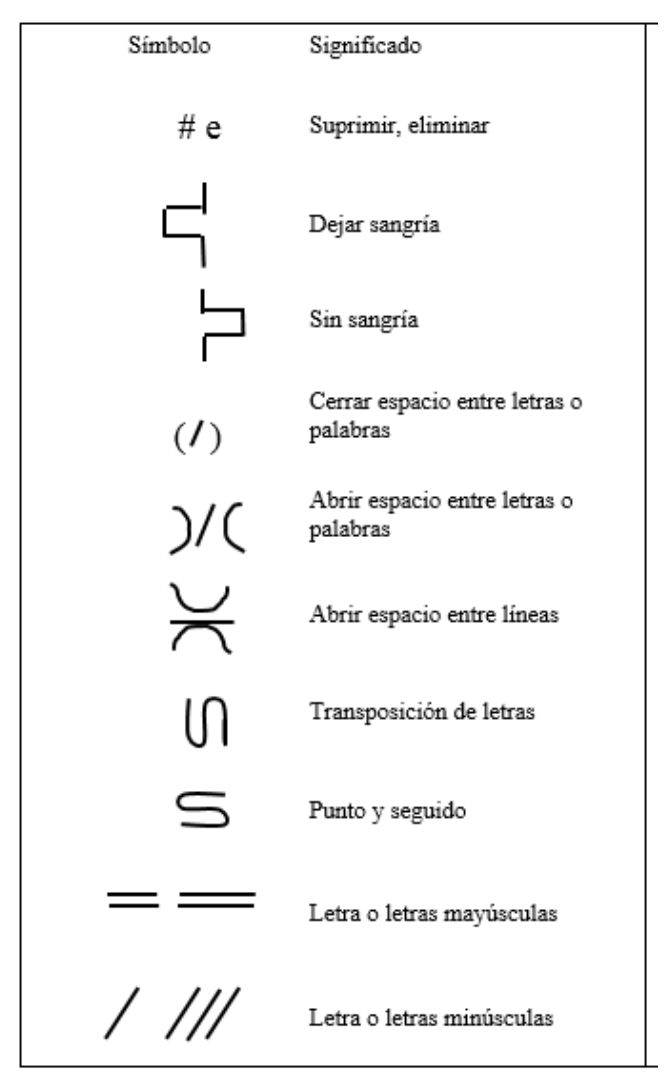

Tabla 1 Símbolos de corrección 


\section{Por qué se deben corregir los originales del trabajo de tesis}

Corregir es un trabajo constante dentro de la investigación del trabajo de tesis y se tiene que hacer para depurar de las imperfecciones el texto escrito, tanto de la forma, como del fondo del contenido. Se debe cuidar el contenido independientemente del área de la ciencia que sea y del nivel de estudios. La finalidad es localizar y señalar las erratas gramaticales y relacionadas con la tipografía (tipo de letra, tamaño de fuente, etc.,) no advertidas. ¿Cuáles pueden ser?

\section{Errores más frecuentes}

a) Verificar la correcta escritura de las palabras. Para realizar esta actividad se requiere una lectura despacio, palabra por palabra, sin confiarse en que el inicio de las letras serán el resultado correcto. No hay que dar por hecho que por las primeras letras, se tiene por completa la palabra. Por ejemplo, al leer juegos, podría decir juevos o la palabra parados, si se encuentra separada sería (para dos), indicará otra significación, para dos personas.

Una letra cambia el significado de una palabra. Por ejemplo, Marco, si nos equivocamos y le anotamos al inicio otra letra, como la $\mathrm{N}$ será muy penoso, ya que las letras $\mathrm{m}$ y $n$ están cerca en el teclado de la computadora. Aunque sea un error de dedo, tiene trascendencia.

b) Imponer mayúsculas a todos aquellos términos que sea propio que las lleven, conforme a las normas establecidas. Las letras altas llamadas mayúsculas tienen la función de resaltar nombres propios y si se anotan en bajas o minúsculas pasan desapercibidas. Por ejemplo en nombre de un lugar, si es Valle Florido y lo anotamos en bajas, simplemente será una característica de lugar. Los nombres se deben respetar en investigaciones históricas, por ejemplo el pirata Laurens o Laurent de Graff, que pasó al conocimiento popular novo hispano como Lorencillo y que aun en la actualidad así se identifica; o William Parker que se le llamó Guillermo Parque y así quedó consignado; y así se pueden agregar muchos ejemplos, nombres de batallas, ríos, valles, minas, etc.
En investigaciones científicas se deben cuidar los nombres de autores. El mexicano es muy ingenioso, a los lugares les cambian las letras, tenemos que verificar que están correctos, bien escritos. Por ejemplo: rumbo a Arandas (Jalisco) existe un letrero que indica los kilómetros para llegar, pero le agregaron algunas letras y decía Parrandas y si lo citamos así en nuestra tesis, todo el valor de la investigación perdería valor.

c) Analizar eficientemente la función que las palabras desempeñan en las oraciones para corregir las utilizadas inadecuadamente. Esta parte es muy importante, aquí radica la fuerza del lenguaje que debe tener en la tesis, dónde ubicar el orden de las palabras. Por ejemplo, cambia la forma de ordenar las palabras, si decimos la investigación de tesis y anotamos la tesis de investigación, es diferente sin duda, pero existen párrafos muy grandes en que decimos la persona que realiza la acción hasta el final y eso ocasiona la falta de claridad en la oración. El sustentante de una tesis tiene que mostrar en su redacción seguridad, si se señala yo creo, tal vez, quizá, se tendrían que cambiar por considero que, es necesario, etc. unifica.

El estilo es personal no se corrige sólo se

d) Procurar que la redacción permita la interpretación más clara y exacta de las ideas expuestas en el escrito, cuidando los giros del lenguaje, la armonía, la concordancia y evitando la redundancia y el pleonasmo. La repetición de palabras en un espacio reducido denota pobreza del lenguaje y se deben anotar sinónimos. La armonía del lenguaje significa que cada uno de nosotros tenemos un lenguaje propio, un estilo, y debemos utilizarlo de esa manera, no podemos intentar con un lenguaje ya expresado por otro escritor, aunque sea exitoso. Cada persona tiene una forma de decir las cosas, unos necesitamos más palabras para expresarnos, otros le damos vuelta al asunto y no somos directos. Se recomienda el lenguaje directo.

e) Usar adecuadamente sinónimos, parónimos, antónimos y homónimos. Tiene relación con la anterior recomendación. 
f) Señalar en el renglón apropiado las sangrías. Se tiene que seguir una forma de presentar la información, no se puede agregar a un párrafo sangría y a otros no, tiene que haber un estilo a seguir, una justificación, por ejemplo, un estilo es no dejar sangría al primer párrafo después de un título, subtítulo o subinciso y en los demás sí. No dejar sangría después de transcripciones de más de cinco líneas, etc. Resulta importante consultar las guías de corrección de editoriales o de periódicos, algunas instituciones tienen sus propias reglas.

g) Consultar con frecuencia el diccionario para aclarar todas las dudas que éste pueda resolver. En una buena corrección, la consulta de un diccionario es frecuente para ver la forma de escribir las palabras, así como para ver el origen de la palabra o término. Nunca terminamos de consultar el diccionario. Se recomienda su uso constante y más para el alumno que está revisando su tesis: en el ámbito jurídico en la actualidad es importante distinguir entre lesiones como un delito o si lo anotamos con c, lecciones será una enseñanza, etc. En el caso de los apellidos generalmente no se les anota acento, por descuido. No es lo mismo leer con acento Martínez, Gómez, Hernández, etc., que sin acento. Pero también se deben respetar conforme están anotados en las actas de nacimiento.

h) Verificar que la puntuación del original sea la misma. La puntuación significa pausa o silencio, se recomienda que los párrafos no sean muy extensos, ocasiona dificultad en su interpretación. En el momento de revisión de la tesis, el original debe estar de acuerdo con el texto anterior original.

i) Revisar que el uso de la sangría sea el indicado. Es importante decir que el uso de sangrías va de acuerdo a los lineamientos de titulación de nuestra institución. Muchas veces nos entregan una guía de cómo presentar el original y debemos tomarla muy en cuenta.

j) Establecimiento de márgenes y jerarquizaciones. Tenemos que fijar los márgenes que tendrá nuestro escrito por cada lado, superior, inferior, derecho e izquierdo. Se recomienda dejar un centímetro más para el lugar donde se empastará el documento.
De igual manera es conveniente inmediatamente leer el documento guía de nuestra institución educativa. (Anderson, Jonathan, Durston, Berry W., 1984, pp. 77-78). Por lo general la presentación de un original lo recomiendan en 12 puntos, ya sea letra Arial o Times New Roman y uno punto cinco de interlínea. Al espacio que separa una línea de otra se le conoce como interlínea.

k) Cuidando que los nombres y las fechas sean las correctas. Muy importante es revisar las fechas, nombres, recurriendo al documento de donde se citan e incluso a otros documentos.

\section{Acentuación}

Escribir correctamente es algo que se aprende poco a poco, con el tiempo y la dedicación. Por medio de esta ponencia podrá servir para darle importancia. Existen algunos errores que el alumno investigador comete en forma frecuente. El uso del acento es muy importante, incluso las letras mayúsculas llevan acento. He observado en mis alumnos que sus errores son por desconocimiento, falta de observación y de consulta del diccionario.

\section{Errores más frecuentes de uso del acento}

\section{De conocimiento}

Son más frecuentes la falta de acento en las palabras que lo llevan que los que se agregan donde no debe ir. Es muy constante que no se anota el acento en:

Más, de cantidad. Sé, del verbo saber. Él, pronombre personal. Mí, pronombre personal. Tú, pronombre personal. Dé, del verbo dar. Sí, de afirmación. Té, de planta. Por qué, el porqué, de interrogación. Aún, todavía. Éste, ésta, éstos, éstas. Pronombres demostrativos que sustituyen una cosa o idea. Qué, pronombre demostrativo. Aquél, aquélla, aquéllos, aquéllas, pronombres demostrativos.

Cuando existen ambigüiedades.

Ésa, ése, ésos, ésas, adjetivos demostrativos, que sustituyen una idea,

\section{Cuando existen ambigüedades.}

Difícilmente, adverbios terminados en mente, conservan el acento. 


\section{Casos en que se anota acento donde no lo lleva. Por ejemplo:}

Fe, dio, vio, fue, ya no llevan acento.

Uso del guión, es muy frecuente el uso del guión para ennumerar.

\section{Lectura de corrección}

Se debe revisar el texto minuciosamente hasta que no se encuentren errores, por lo general tres veces o más si es necesario. Recomiendo solicitar la ayuda de otra persona para que lea el documento con las correcciones y de esa manera el alumno investigador verifica que se haya hecho el cambio en forma clara y correcta. Se debe leer como si el autor fuera otra persona, ya que podría dar por ciertos muchos datos de memoria. (Zubizarreta, Armando, 1984, p. 194), (Reyes Coria, Bulmaro, 1986, p. 96).

\section{Unificación del original}

Unificación, es anotar en forma completa las partes de una tesis, que lleven el mismo orden. Este trabajo representa mucho tiempo y no se le da el valor que tiene. ¿Qué se debe unificar?

1. Que todas las páginas lleven número. A veces a las páginas principales no se les anota página, pero todas cuentan. Generalmente la tesis tiene la información por la parte frente de la hoja, pero cada parte es página (frente y vuelta). La falta de número de página es muy frecuente en trabajos escolares o que la numeración de la tesis inicie después de la portada interna. El número deberá ser en el mismo lado, no unas páginas en la parte superior y otras en el lado inferior. Se recomienda anotar el número de página en la parte superior derecha porque una tesis tiene fines didácticos, de constante revisión.

2. Orden de los datos de una referencia y que se encuentren completos. Se recomienda que se respete el orden que se ha elegido para anotar, las partes de una referencia. El sistema puede ser APA o a pie de página, pero al final lleva las fuentes utilizadas y ahí no debe faltar ningún dato de la obra. La redacción debe llevar autor o autores, obra con subtítulo o subtítulos, editorial, lugar de impresión de la obra, página o páginas. En qué orden, de la manera que es recomendada por la institución donde se estudia. Si no existe un manual de presentación de la tesis, entonces se pueden consultar diversas obras de metodología e incluso la propuesta por el autor de esta ponencia. (Orozco Orozco, 2018).

Existen diversas formas de redactar las referencias, algunas piden que ya no se anote la palabra editorial, solo el nombre, etc.

Mi propuesta de anotar las referencias bibliográficas es un número pequeño realzado llamado número voladito, en el final de párrafo y a pie de páginas los datos, ya que de esta manera se revisa la información inmediatamente. Por ejemplo:

1 Orozco Orozco, José Zócimo, Cómo planear y desarrollar tesis en posgrado de Derecho, Acento, Guadalajara, Jalisco, México, 2018, pp. 166-1467.

3. Anotar en forma completa los nombres de personas y de organizaciones, instituciones, etc., que se citan por primera vez. En los siguientes párrafos podrán mencionarse parte de ellos. Por ejemplo, el nombre de una autor, de una organización (a menos que haya un índice al principio de la tesis). Por ejemplo: Reyes Coria, Bulmaro, Organización de los Estados Americanos (OEA).

4. Acomodo de las partes de la tesis. La tesis debe tener todas sus partes, que no falten las conclusiones y de ser posible las propuestas. Debemos anotar el número de capítulo como lo hicimos en dicho capítulo inicial y los siguientes en la misma forma elegida. Los capítulos se pueden anotar el número con letra, número romano o número arábigo. Es el mismo caso de la investigación si está separada también por partes.

5. Las transcripciones. Verificar que toda transcripción que pase de cinco líneas, se haga aparte del párrafo, con menos espacio, en menos puntos y que el siguiente párrafo inicie sin sangría.

6. Forma de dejar la sangría al inicio de cada párrafo. Cuidar que la forma de presentar la información de dejar sangría sea igual en cada párrafo. También debemos cuidar que los párrafos no sean demasiado grandes. Una página sin separación de párrafos es muy pesada su lectura y no motiva al director y sinodales de tesis su lectura. 
7. Tipo y tamaño de letra. Verificar que el tamaño de letra sea el mismo, así como el tipo de letra, en toda la investigación. Por lo general son 12 puntos y la letra Arial o Times New Roman, dependiendo de la institución donde se realizan los estudios. En los títulos de partes y capítulos se recomienda en 14 o 16 puntos.

8. Márgenes. Debe llevar el documento los mismos márgenes: izquierdo y derecho de 3 centímetros, superior e inferior de 2.5 centímetros.

9. Unificación de números de cuadros, esquemas, dibujos, mapas, tablas, fotografías, etc. Cuidar el orden de la numeración de estos elementos, número ascendente, anotar el nombre del elemento.

10. Evitar repetición de palabras en un espacio muy seguido. Es mejor utilizar sinónimos o referirnos con un lenguaje diferente para evitar repeticiones.

11. El tiempo en que se escribe la tesis. No se escribe al director. (Eco, Umberto, 1996, p. 178). Es recomendable redactar en forma impersonal. (Domínguez, Silvia; Sánchez, Enrique E. y Sánchez, Gabriel, A., 2009, pp. 78). Es mejor en general, que en forma personal, yo hice, yo digo, etc. En el primer capítulo de marco teórico conceptual se puede hablar en pasado o se puede utilizar el tiempo histórico presente en los antecedentes.

12. Quitar en enumeraciones el guion. Por ejemplo: 1.-, mejor 1. También se debe evitar el abuso de letras mayúsculas cuando no sea necesario.

\section{Fuentes para la corrección de la tesis}

1. Diccionario de lenguaje. Puede ser el Diccionario de la Real Academia Española. Se localiza electrónicamente por lo que se puede consultar de manera instantánea, al momento de la duda.

2. Diccionario del área del conocimiento de la tesis.

Reyes Coria, Bulmaro, (2008). Metalibro, manual del libro en la imprenta, Universidad Nacional Autónoma de México, Dirección General de Publicaciones y Fomento Editorial, México.
Reyes Coria, Bulmaro, (1986). Manual de estilo editorial, Limusa, México.

Zavala Ruiz, Roberto, (1995). El libro y sus orillas, tipografía, originales, redacción, corrección de estilo y de pruebas, Universidad Nacional Autónoma de México, Biblioteca del Editor, Dirección General de Fomento Editorial, México.

Alonso, Martín, (1979). Ciencia del lenguaje y arte del estilo, Madrid, España.

Martínez de Souza, José, (1995). Diccionario de tipografia y del libro, Paraninfo, Madrid, España.

Orozco, José Zócimo, (2018). Cómo planear y desarrollar tesis en posgrado de Derecho, Acento, Guadalajara, Jalisco, México.

3. Fuentes de la información de la tesis. Las obras consultadas o copias de las partes principales, para verificar información y hacer la redacción de referencias al final de la tesis.

4. Archivo de documentos sobre trámites de titulación. Protocolo, nombramiento de director, recomendaciones en coloquios sobre el avance de la investigación, pagos por trámites de titulación, aprobación de protocolo, director de tesis, lectores, etc.

\section{Conclusiones}

La revisión de tesis en forma minuciosa es necesaria a fin de lograr tesis de calidad y una mejor calificación en su evaluación.

Es importante que el director instruya desde el principio al alumno en su preparación para hacer una mejor revisión de su trabajo de tesis.

\section{Propuestas}

Primera. Se debe fortalecer el conocimiento del alumno para la revisión de tesis. Lo conveniente es que la institución apoye con pláticas o conferencias al inicio de cada ciclo escolar y que el director de tesis verifique que se apliquen esos conocimientos por parte del alumno. Con esto se logrará dar pasos sólidos en la construcción del conocimiento científico del alumno. 
Segunda. Que la revisión llegue hasta el mismo examen de titulación y las recomendaciones que se hacen por los integrantes del jurado se agreguen al original de tesis.

Las observaciones que se hacen en el examen son muy valiosas y no deben quedarse en el olvido, sin integrarse al documento que será consultado por alumnos que desean titularse o por otros investigadores.

Es el momento cumbre donde se hacen las mejores indicaciones de lo que la tesis debió comprender, de lo que le hizo falta, de cómo se hubiera logrado la tesis perfecta, es cuando se expresa con mayor honestidad lo que piensa de la tesis.

La forma de integrarse puede ser como un escrito realizado por los sinodales, indicando las recomendaciones hechas al documento de titulación o pedirle al alumno que las integre al documento y lo regrese a la institución.

Tercera. El director de tesis debe tener tiempo para dedicarle al alumno; es imprescindible que el alumno tenga la confianza de contactar a su director ante cualquier duda que surja a lo largo de la investigación para recibir la orientación correcta y de esta manera no perder tiempo haciendo trabajo infructuoso.

\section{Referencias}

Anderson, Jonathan, et., al. (1984). Redacción de tesis y trabajos escolares, Diana,

Domínguez, Silvia; Sánchez, Enrique E. y Sánchez, Gabriel, A. (2009). Guía para elaborar una tesis, Mc Graw Hill, México.

Eco, Umberto. (1996). Cómo se hace una tesis, técnicas y procedimientos de investigación, estudio y escritura, Gedisa, España.

Estelle M. Philis, Derek S. Pugh. (2008). La tesis doctoral, Profiy, Barcelona,

Orozco Orozco, José Zócimo. (2018). Cómo planear y desarrollar tesis en posgrado de Derecho, Acento, Guadalajara, Jalisco, México
Reyes Coria, Bulmaro. (2008). Metalibro, manual del libro en la imprenta, Universidad Nacional Autónoma de México, Dirección General de Publicaciones y Fomento Editorial, México.

Reyes Coria, Bulmaro. (1996). Manual de estilo editorial, Limusa, México.

Rivera-Camino, Jaime. (2014). Cómo escribir y publicar una tesis doctoral, Ecic, Madrid, España.

Vivaldi, G. Martín, C. (1998). Curso de redacción, teoría y práctica de la composición y del estilo, Madrid, España.

Zubizarreta, Armando. (2004). La aventura del trabajo intelectual, cómo estudiar e investigar, Addison-Wesley Iberoamericana, Estados Unidos. . 